\title{
Quantum Phase Transitions and the Breakdown of Classical General Relativity
}

\author{
G. Chapline \\ Los Alamos National Laboratory, Los Alamos, New Mexico 87545 \\ E. Hohlfeld, R. B. Laughlin, and D. I. Santiago \\ Department of Physics, Stanford University, Stanford, California 94305
}

(May 29, 2018)

\begin{abstract}
It is proposed that the event horizon of a black hole is a quantum phase transition of the vacuum of space-time analogous to the liquid-vapor critical point of a bose fluid. The equations of classical general relativity remain valid arbitrarily close to the horizon yet fail there through the divergence of a characteristic coherence length $\xi$. The integrity of global time, required for conventional quantum mechanics to be defined, is maintained. The metric inside the event horizon is different from that predicted by classical general relativity and may be de Sitter space. The deviations from classical behavior lead to distinct spectroscopic and bolometric signatures that can, in principle, be observed at large distances from the black hole.
\end{abstract}

PACS numbers: 04.70.Dy,05.70.Jk,05.30.Jp,64.60.Ht

\section{INTRODUCTION}

Quantum mechanics is incompatible with classical general relativity. While there are many ways of articulating the problem, all reduce in the end to the absence of universal time required for the many-body Schrödinger equation

$$
i \hbar \frac{\partial \Psi}{\partial t}=\mathcal{H} \Psi
$$

to make sense. This equation is the logical underpinning of quantum field theory and statistical mechanics, and thus of our microscopic understanding of the entire natural world outside gravity. General relativity predicts that certain stars evolve at the end of their lives into black holes characterized by surfaces at which time, as measured by a clock at infinity, stands still. Gravity is well-behaved at this surface, in that a free-falling observer passes through in finite proper time without being ripped apart by tidal forces, but quantum mechanics is not. The paradox is fundamental. It has led to proposals for revising the laws of quantum mechanicst and to speculations that black holes may destroy quantum informatione.

In this paper we propose a resolution of this problem that is fully quantum-mechanical and is based on principles that can be tested in the laboratory. The essence of the idea, illustrated in Fig. 1, is that the black hole event horizon is a continuous quantum phase transition of the vacuum of space-time roughly analogous to the quantum liquid-vapor critical point of an interacting bose fluid. In such systems the classical description of the "vacuum" on either side of the horizon fails on length scales smaller than a characteristic length $\xi$, a quantummechanical quantity, that diverges at the horizon. The classical equations remain exactly valid up up to the horizon, but only in context of a special, unphysical order of limits. In a real experiment done at finite size the diverging length $\xi$ will eventually reach this size and cause the classical description of the experiment to fail. In the bose fluid the approach to the critical surface is signaled by the vanishing of the speed of sound. In a black hole the approach to the horizon is signaled by the vanishing of the time dilation factor. An apt analogy between the two thus requires the time dilation to increase inside the event horizon-at odds with the prediction of classical general relativity.

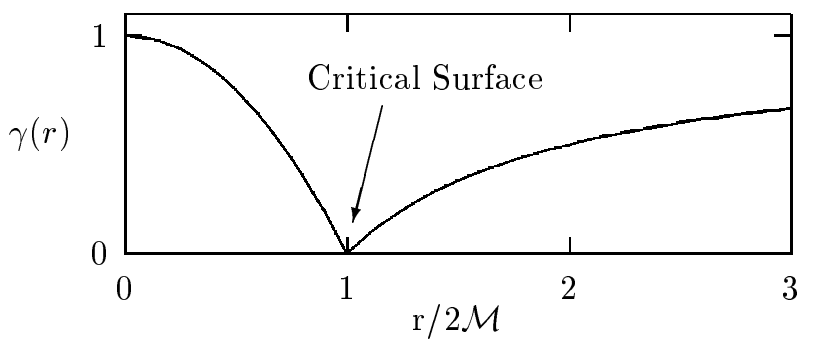

FIG. 1. Prototype time dilation factor $\gamma(r)$ in the vicinity of a black hole event horizon.

The notion that general relativity might be an emergent property in a condensed-matter-like quantum theory of gravity has a long historyl. In 1968 Sakharov observed that space-time in Einstein gravity was similar to to stressed mattert. In Sakharov's model there is no order parameter similar to that in superfluids, but one with such order parameters has been proposed 1 . In 1982 Unruh observed a close analogy between sound propagation in background hydrodynamic flow and field propagation in curved space-time. Following Unruh's lead, models for a black hole based on superfluid flow of ${ }^{3} \mathrm{He}$ and atomic bose condensates $\mathrm{l}$ have been put forward. Mohazzab has recently proposed an analogy between black hole event horizons and the normal-superfluid interface 9 of ${ }^{4} \mathrm{He}$. Ueda and Huang have noted the similarity be- 
tween black hole collapse and the ipstability of atomic bose condensates to attractive forces 10 .

However, our proposal differs from this recent work in the key respect that it ascribes black hole behavior at the event horizon to a quantum ground state. This enables us to argue for the first time that collective effects are the correct explanation for the puzzling behavior of black holes-and by implication the apparent incompatibility of quantum mechanics and general relativity.

\section{BOSONIC MATTER}

The simplest kind of matter is ${ }^{4} \mathrm{He}$ and substances like it11. It is a collection of $N$ atoms obeying Eq. (1) with

$$
\mathcal{H}=-\frac{\hbar^{2}}{2 M} \sum_{j=1}^{N} \nabla_{j}^{2}+\mathcal{V}\left(\mathbf{r}_{1}, \ldots, \mathbf{r}_{N}\right)
$$

subject to the condition that $\Psi$ be symmetric under interchange of any two of its arguments $\mathbf{r}_{1}, \ldots, \mathbf{r}_{N}$. The ground state is the energy eigenstate

$$
\begin{gathered}
\Psi\left(\mathbf{r}_{1}, \ldots, \mathbf{r}_{N}, t\right)=e^{-i E_{0} t / \hbar} \Phi\left(\mathbf{r}_{1}, \ldots, \mathbf{r}_{N}\right) \\
\mathcal{H} \Phi=E_{0} \Phi
\end{gathered}
$$

with the lowest eigenvalue $E_{0}$. The zero-temperature equation of state of the matter is the functional dependence of $E_{0}$ on various parameters in the hamiltonian, such as the confinement volume $V$ or atomic mass $M$. This dependence is smooth and continuous except at quantum phase transitions, where it is singular.

The properties of ${ }^{4} \mathrm{He}$ demonstrate that both zerotemperature phasefransitions of bosonic matter and the liquid phase exist 12 . ${ }^{4} \mathrm{He}$ is a solid at pressures above 25 atmospheres and zero temperature. As the pressure is dropped below 25 bar it melts into a liquid with unmeasurably small quantum vapor pressure-meaning that it puddles at the bottom of a container larger than itself and will not evaporate at zero temperature. This liquid, like all the fluids we will consider here, is much colder than the bose-einstein condensation temperature and is thus a pure superfluid.

The vapor phase of bosonic matter also exists in nature in the newly-discovered "bose-einstein condensates"-a name that is somewhat misleading as these systems exhibit a finite sound speed11. They are also metastable excited states rather than ground states, and are more aptly called supersaturated quantum vapors. Their behavior is fully consistent with Bogoliubov's original description of superfluid broken symmetry in ${ }^{4} \mathrm{He}$, which was based on weak repulsive potentials and was actually a description of the quantum gas 13 .

The nature of the zero-temperature liquid-vapor transition in these systems is, however, controversial. In 1977
Miller, Nosanow and Parish 14 performed a realistic variational study of lennard-jones fluids and found that the critical point could not be reached by varying pressure. They concluded from this that bose fluids never have a conventional critical point. However there is no general principle leading to that conclusion, and mere recent studies based on different model assumptions 15 find behavior more consistent with that of classical fluids. We will proceed on the assumption that the models predicting a conventional critical point were solved correctly, and that the result of Miller, Nosanow and Parrish was specific to the class of model they were studying.

The quantum liquid-vapor transition, or something like it, may have been seen experimentally in these condensates 16.17. The relevant experiments exploit hyperfine scattering resonances in certain isotopes to tune the s-wave phase shift through zero by means of a magnetic field. When Cornish et al 17 did this with ${ }^{85} \mathrm{Rb}$ they found the ball of vapor to first contract - as expected if its pressure were being reduced - and then explode partially, leaving a remnant condensate with a "halo" of hot gas. Interpretation of this effect as a phase transition is complicated by the metastable nature of the condensate and increased rate of recombination into the true ground state that occurs at high densities. However it occurs abruptly at the place where such a transition is expected and is preceded by a dramatic softening of the compressibility.

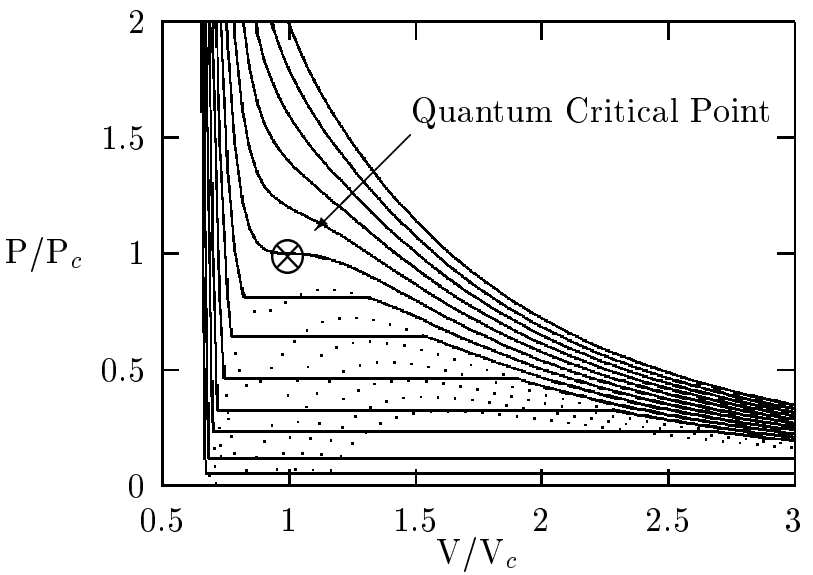

FIG. 2. Phenomenological equation of state defined by Eq. (6) for various values of the parameter $c$ near the critical value. The dotted lines indicate the Maxwell loops.

\section{MODEL HAMILTONIAN}

Let us now proceed to construct a model for the quantum liquid-vapor transition. The simplest realization of this transition in classical fluid is the Van der Waals equation of state 18

$$
(V-b)\left(P+\frac{a}{V^{2}}\right)=N k_{B} T .
$$


By analogy with this let us consider the phenomenological quantum equation of state

$$
\left(V^{2}-b\right)\left(P+\frac{a}{V^{4}}\right)=c,
$$

shown in Fig. 2. This is generated in the mean-field approximation from the field theory

$$
\mathcal{L}=\psi^{*}\left(i \hbar \frac{\partial}{\partial t}+\mu\right) \psi-\frac{\hbar^{2}}{2 M}|\nabla \psi|^{2}-\mathcal{U}\left(|\psi|^{2}\right),
$$

with $|\psi|^{2}$ interpreted as the density $\rho=N / V$ and

$$
\mathcal{U}=\frac{c}{2 \sqrt{b} V} \ln \left(\frac{V+\sqrt{b}}{V-\sqrt{b}}\right)-\frac{a}{3 V^{4}} .
$$

After canonical quantization this becomes equal to Eq. (2) with a short-range multiconfigurational potential $\mathcal{V}$. At zero temperature this system exhibits the phenomenon of bose condensation-i.e. acquires a superfluid order parameter $\psi$ with low-energy dynamics described by the extremal condition

$$
i \hbar \frac{\partial \psi}{\partial t}=-\frac{\hbar^{2}}{2 M} \nabla^{2} \psi+\left[\mathcal{U}^{\prime}\left(|\psi|^{2}\right)-\mu\right] \psi .
$$

This is the Gross-Pitaevskii equation 19 . The particle density and current density, defined by

$$
|\psi|^{2}=\rho \quad \rho \mathbf{v}=\frac{\hbar}{2 M i}\left(\psi^{*} \nabla \psi-\psi \nabla \psi^{*}\right),
$$

then satisfy hydrodymamic conservation of particle number and momentume

$$
\frac{\partial \rho}{\partial t}+\nabla \cdot(\rho \mathbf{v})=0 \quad M \frac{\partial}{\partial t}(\rho \mathbf{v})+\nabla P=0 .
$$

The quiescent state of the fluid is described by the uniform solution $\psi_{0}$ satisfying

$$
\mathcal{U}^{\prime}\left(\left|\psi_{0}\right|^{2}\right)-\mu \psi_{0}=0 \quad\left(P V+E_{0}=\mu\right) .
$$

The particle density is fixed by suitably adjusting the chemical potential $\mu$. Small perturbations to this solution

$$
\psi=\psi_{0}+\delta \psi_{\mathrm{R}}+i \delta \psi_{\mathrm{I}}
$$

then satisfy

$$
\begin{gathered}
\hbar \frac{\partial\left(\delta \psi_{R}\right)}{\partial t}=-\frac{\hbar^{2}}{2 M} \nabla^{2}\left(\delta \psi_{\mathrm{I}}\right) \\
-\hbar \frac{\partial\left(\delta \psi_{\mathrm{I}}\right)}{d t}=-\frac{\hbar^{2}}{2 M} \nabla^{2}\left(\delta \psi_{\mathrm{R}}\right)+\frac{2 B}{\rho}\left(\delta \psi_{\mathrm{R}}\right),
\end{gathered}
$$

to linear order and thus give the dispersion relation

$$
\hbar \omega_{q}=\sqrt{\left(\hbar v_{s} q\right)^{2}+\left(\frac{\hbar^{2} q^{2}}{2 M}\right)^{2}}
$$

for compressional sound. This identifies $\xi=\hbar / M v_{s}$ as the length scale for the failure of hydrodynamics. This same scale appears is the Bogoliubov solution 13 .

As usual the region of negative compressibility is an inaccurate description of liquid-gas phase separation and is replaced with a Maxwell construction. This is discussed more thoroughly in Appendix A. For this reason there is one and only one point in the diagram where the bulk modulus $B=-V(\partial P / \partial V)$ is zero, namely the critical point.

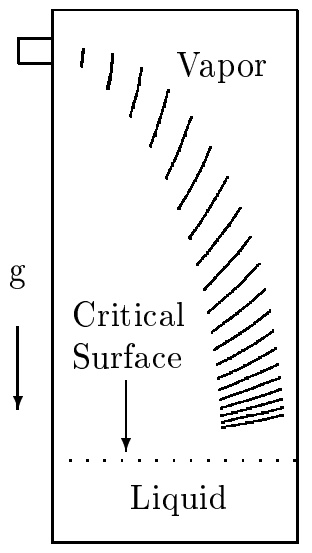

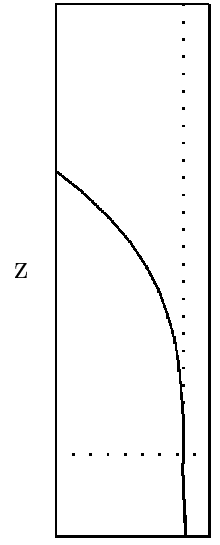

$\mathrm{P}$

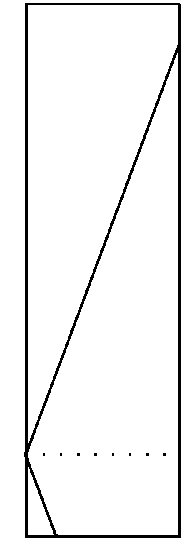

$\mathrm{v}_{s}$
FIG. 3. Illustration of thought experiment in which pressure increases toward the bottom of a tank of quantum fluid. Sound emitted from a transducer on the side of the tank is refracted downward toward the critical surface where the sound speed collapses to zero. The wave fronts shown are for a solution of Eq. 20) with the pressure and sound speed profiles given by Eq. (25) and plotted on the right. The quantum pathologies in this case are exactly the same as those at a Schwarzschild black hole.

\section{CRITICAL SURFACE EVENT HORIZON}

Let us now imagine a thought experiment, illustrated in Fig. 3, in which a tall tank on the surface of the earth is filled with a quantum fluid characterized by a critical equation of state. The pressure increases toward the bottom of the tank due to gravity and at some critical depth reaches, and then surpasses, the critical pressure. Sound waves are refracted toward this surface just as light is refracted toward a black hole horizon and for the same reason, namely that the propagation speed measured by a clock at infinity vanishes there. For the specific equation of state defined by Eq. (6) with $c=8 a / 27 b$, which reduces near the critical point to

$$
\rho_{c}=\frac{1}{\sqrt{3 b}} \quad P_{c}=\frac{a}{27 b^{2}} \quad v_{c}=\sqrt{\frac{P_{c}}{M \rho_{c}}}
$$

$$
\frac{P}{P_{c}}-1 \simeq 12\left(\frac{\rho}{\rho_{c}}-1\right)^{3},
$$


we have

$$
\frac{P}{P_{c}} \simeq 1-\frac{g z}{v_{0}^{2}} \quad \frac{v_{s}}{v_{0}} \simeq 6\left|\frac{g z}{12 v_{0}^{2}}\right|^{1 / 3} .
$$

near the critical surface at $z=0$. Small density fluctuations $\rho \rightarrow \rho+\delta \rho$ then propagate according as

$$
\nabla \cdot\left[v_{s}^{2} \nabla(\delta \rho)\right]=\frac{\partial^{2}(\delta \rho)}{\partial t^{2}}
$$

This is qualitatively the same as the scalar wave equation

$$
\nabla \cdot\left[v_{s} \nabla \phi\right]=\frac{1}{v_{s}} \frac{\partial^{2} \phi}{\partial t^{2}}
$$

one obtains from

$$
\frac{\partial}{\partial x^{\mu}}\left(\sqrt{-g} g^{\mu \nu} \frac{\partial \phi}{\partial x^{\nu}}\right)=0
$$

using the gravitational metric

$$
d s^{2}=g_{\mu \nu} d x^{\mu} d x^{\nu}=d x^{2}+d y^{2}+d z^{2}-v_{s}^{2} d t^{2} .
$$

The particular power law with which the sound speed vanishes in this experiment is not important and can easily be modified. For example one can imagine weakening the downward force on the atoms according to the rule

$$
g=g_{0}\left(1-e^{-z^{2} / \ell^{2}}\right)
$$

so that

$$
\frac{P}{P_{c}} \simeq 1-\frac{g_{0} z^{3}}{3 \ell^{2} v_{0}^{2}} \quad \frac{v_{s}}{v_{0}} \simeq 6\left|\frac{g_{0} z^{3}}{36 \ell^{2} v_{0}^{2}}\right|^{1 / 3}
$$

The analogy with gravity is more obvious in this case because the metric just outside the event horizon of a Schwarzschild black hole can be written in this form with $v_{s}=c^{3} z / 4 G M$.

\section{QUANTUM-CRITICAL DISSIPATION}

In contrast to the case of classical gravity, however, the paradoxes of sound propagation near the critical surface have a simple quantum-mechanical resolution: Sound ceases to make sense near the horizon because the principles of hydrodynamics fail on length scales smaller than the correlation length $\xi=\hbar / M v_{s}$ and time scales longer than $\xi / v_{s}$. At the horizon both diverge to infinity. A sound quantum with fixed frequency $\omega$ propagating toward the horizon reaches the point at which $\omega \geq v_{s} / \xi$ in finite time and decays there into the soft excitations of the critical point. These are dense, so most of the energy thermalizes. This effect has never been observed experimentally, but its classical analogue, critical opalescence, is well knpwn and has been studied extensively by light scattering 21 .
Let us now consider this effect in detail. At the critical point the Lagrangian is effectively

$$
\mathcal{L}_{\text {eff }}=\psi^{*}\left(i \hbar \frac{\partial}{\partial t}-\mu\right) \psi-\frac{\hbar^{2}}{2 M}|\nabla|^{2}-\frac{3 P_{c}}{\rho_{c}^{2}}\left(|\psi|-\psi_{0}\right)^{4}
$$

i.e. a nonrelativistic bose gas with a high-order nonlinearity. The corresponding quantum Hamiltonian is

$$
\mathcal{H}_{\mathrm{eff}}=\sum_{\mathbf{q}} \frac{\hbar^{2} q^{2}}{2 M} a_{q}^{\dagger} a_{q}+\frac{3 P_{c} \psi_{0}^{4}}{V \rho_{c}^{2}} \sum_{\mathbf{q}_{1} \mathbf{q}_{2} \mathbf{q}_{3} \mathbf{q}_{4}}
$$

$$
\times \delta\left(\mathbf{q}_{1}+\mathbf{q}_{2}+\mathbf{q}_{3}+\mathbf{q}_{4}\right)\left(a_{\mathbf{q}_{1}}+a_{-\mathbf{q}_{1}}^{\dagger}\right)\left(a_{\mathbf{q}_{2}}+a_{-\mathbf{q}_{2}}^{\dagger}\right)
$$

$$
\times\left(a_{\mathbf{q}_{3}}+a_{-\mathbf{q}_{3}}^{\dagger}\right)\left(a_{\mathbf{q}_{4}}+a_{-\mathbf{q}_{4}}^{\dagger}\right)
$$
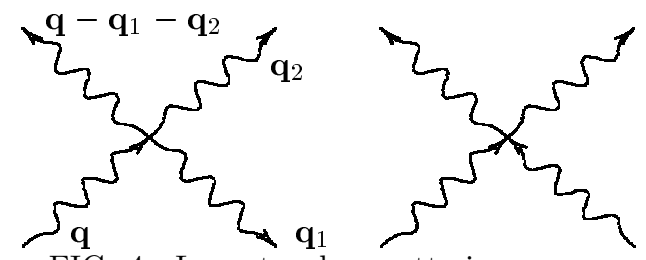

FIG. 4. Lowest-order scattering processes in the critical region. The process on the left causes mass renormalization and decay at zero temperature. The one on the right causes critical opalescence.

The important decay and scattering processes are shown in Fig. 4. The first correction to the the particle selfenergy renormalizes $M$ and gives an imaginary part

$$
\operatorname{Im} \Sigma_{\mathbf{q}}(\omega)=\left(\frac{3 P_{c}}{\rho_{c}^{2} V}\right)^{2} \sum_{\mathbf{q}_{1} \mathbf{q}_{2}} \operatorname{Im}[\hbar \omega
$$

$$
\left.-\frac{\hbar^{2}}{2 M}\left(\left|\mathbf{q}_{1}\right|^{2}+\left|\mathbf{q}_{2}\right|^{2}+\left|\mathbf{q}_{1}+\mathbf{q}_{2}+\mathbf{q}\right|^{2}\right)+i \eta\right]^{-1}
$$

$$
=-\frac{3}{16 \pi^{2}}\left(\frac{M}{\hbar^{2}}\right)^{3}\left(\frac{P_{c}}{\rho_{c}^{2}}\right)^{2}\left(\hbar \omega-\frac{\hbar^{2} q^{2}}{6 M}\right)^{2} \Theta\left(\hbar \omega-\frac{\hbar^{2} q^{2}}{6 M}\right) .
$$

The decay rate for a boson of energy $\hbar \omega=\hbar^{2} q^{2} / 2 M$ is thus

$$
\frac{\hbar}{\tau}=\frac{1}{3 \pi^{2}}\left(\frac{M}{\hbar^{2}}\right)^{3}\left(\frac{P_{c}}{\rho_{c}^{2}}\right)^{2}(\hbar \omega)^{2} .
$$

This implies that the free boson becomes more and more sharply defined as the energy is lowered, so that in the low-energy limit one retrieves the ideal noninteracting bose gas 2 .

Let us now consider the several experimental signatures of this effect: 

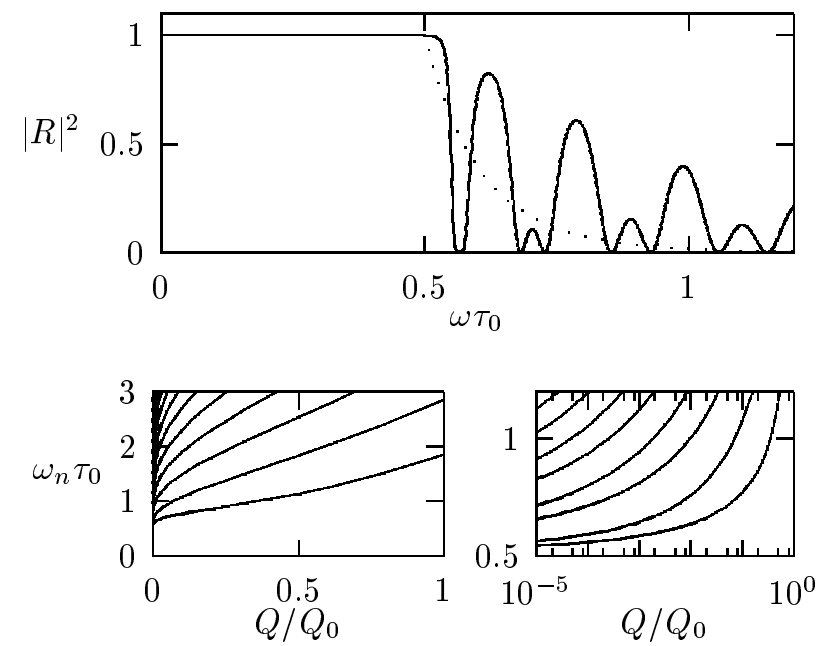

FIG. 5. Top: Reflectivity as a function of $\omega \tau_{0}$ predicted by Eq. (31) for the case of $Q / Q_{0}=10^{-5}$, with $Q_{0}=\left(2 M / \hbar \tau_{0}\right)^{1 / 2}$. The interface thickness is assumed to be of order $1 / Q_{0}$ to make the resonances visible. The dotted line is the $Q \rightarrow 0$ limit described by Eq. (32). Bottom: Dispersion relation of interface bound states plotted both linearly (left) and semi-logarithmically (right).

\section{A. Reflectivity}

At very low frequencies a phonon impinging on the surface at zero temperature is coherently reflected or transmitted depending on its energy. Solving the equation

$$
\hbar^{2} \frac{\partial^{2} \phi}{\partial t^{2}}=\left(\frac{\hbar}{\tau_{0}}\right)^{2} \nabla \cdot\left(z^{2} \nabla \phi\right)-\left(\frac{\hbar^{2}}{2 M}\right)^{2} \nabla^{4} \phi
$$

where $1 / \tau_{0}=\partial v_{s} / \partial z$ (cf. Eqs. (14) and (15) with $\delta \psi_{\mathrm{R}}=$ $\partial \phi / \partial z)$, we obtain the reflection coefficient shown in Fig. 5. The momentum component $Q$ in the plane, which is conserved, acts like a mass and allows the phonon to become trapped at certain energies $\hbar \omega_{n}$. These produce transmission resonances that become narrower and narrower with increasing thickness of the interface. The positions of these resonances depend on $M$ and may thus be used spectroscopically to determine this parameter. For $Q>>Q_{0}$ they occur at the harmonic oscillator values

$$
\hbar \omega_{n} \simeq \frac{\hbar^{2} Q^{2}}{2 M}+\left(n+\frac{1}{2}\right) \sqrt{2} \frac{\hbar}{\tau_{0}} .
$$

Normal incidence $(Q \rightarrow 0)$ is a singular point where the discrete energies collapse to a continuum characterized by the reflectivity

$$
|R|^{2}=\left[\begin{array}{ll}
1 & \omega \tau_{0}<1 / 2 \\
\cosh ^{-2}\left(\pi \sqrt{\left(\omega \tau_{0}\right)^{2}-1 / 4}\right) & \omega \tau_{0}>1 / 2
\end{array}\right] .
$$

This result is discussed further in Appendix B.

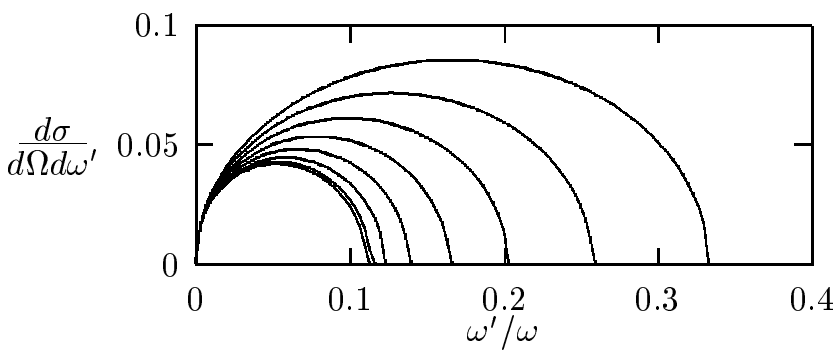

FIG. 6. Differential cross-section given by Eq. (33) for inelastic scattering of sound from a critical surface as a function of scattered frequency $\omega^{\prime}$ for values of $\theta$ ranging from 0 to $\pi / 2$. The maximum value of $\omega^{\prime} / \omega$ for sound reflected normally $(\theta=0)$ is $1 / 9$.

\section{B. Inelastic Scattering}

The horizon is opaque to high-frequency sound waves impinging upon it and inelastically scatters about $1 / 8$ of them back out with a strong red shift. When $\tau_{0} / \tau>1$, where $\tau$ is given by Eq. 29, incoming phonon decays with $100 \%$ probability, and one (but not two) of the three bosons thus generated can escape back out the surface. This gives a differential cross section per unit area $A$ to scatter sound of frequency $\omega$ back in solid angle $d \Omega$ at frequency $\omega^{\prime}<\omega$ of

$$
\frac{d \sigma}{d \Omega d \omega^{\prime}}=\frac{27 A}{16 \pi^{2} \omega} \sqrt{3 x[1-3 x-2 \sqrt{x} \cos (\theta)]},
$$

where $x=\omega^{\prime} / \omega$. This is plotted in Fig. 6. Thus the horizon is a blackbody that fluoresces red. It is an extremely efficient thermalizer of energy, however.

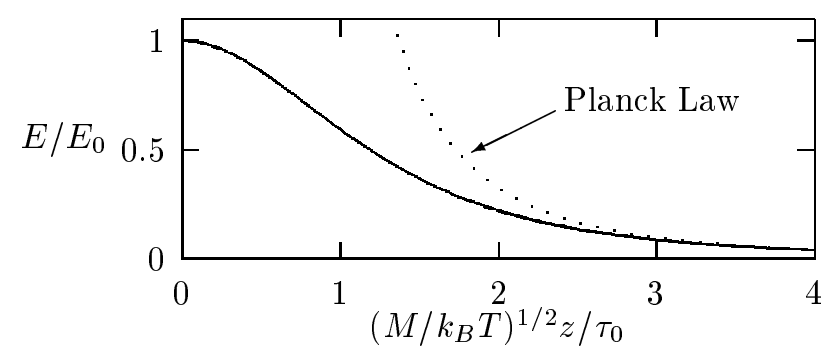

FIG. 7. Thermal energy per unit volume defined by Eqs. (34) and (35) for a critical surface at temperature $T$. The divergent planck law is shown for comparison.

\section{Heat Capacity}

The horizon becomes much more dissipative at low frequencies if the critical region is hot. At temperatures below the bose condensation temperature $\left(k_{B} T_{B} \simeq\right.$ $\left.\hbar^{2} \rho^{2 / 3} / 2 M\right)$ the absorption rate for a phonon of energy $E<<k_{B} T$ is roughly Eq. (29) with $k_{B} T$ substituted for $E$ ( $c f$. Fig. 4). This effect is equivalent to classical critical opalescence. Its heat capacity is large but finite. The energy density a distance $z$ away from the interface is 


$$
\frac{E}{V}=\frac{1}{2 \pi^{2}} \int_{0}^{\infty} \frac{\hbar \omega_{q} q^{2} d q}{\exp \left(\beta \hbar \omega_{q}\right)-1}
$$

where $\omega_{q}$ is given by Eq. (16) with $v_{s}=z / \tau_{0}$. This is plotted in Fig. 7. It may be seen to limit properly as $z \rightarrow 0$ to

$$
\frac{E_{0}}{V}=0.128\left(\frac{M}{\hbar^{2}}\right)^{3 / 2}\left(k_{B} T\right)^{5 / 2} .
$$

Thus the criticality cuts off the divergence in the planck law $E / V=\left(\pi^{2} / 30\right)\left(k_{B} T\right)^{4}\left(\tau_{0} / \hbar z\right)^{3}$.

\section{DIMENSIONAL ANALYSIS}

It is unfortunately not the case that knowledge of the mechanical analogues of $c$ and $G$ is sufficient to determine the correlation lenth $\xi$. The fluid possess a dimensionless parameter $\eta=\xi^{3} \rho$ that cannot be determined by any low-frequency measurement. There is indeed a unique combination of the sound speed $v_{s}$, mass density $M \rho$, and $\hbar$ that has units of length, but this cannot be associated with $\xi$ without assuming that $\eta=1$, which need not be the case.

The analogue of Newton's constant $G$ in the fluid is the inverse mass density $1 / M \rho$. There is no inverse-square attraction between two masses in the superfluid because its monopolar nature makes this interaction shortranged. However, we can sensibly compare the radiation produced by rotating quardupoles. A pair of masses $\mathcal{M}$ orbiting around each other at separation $\ell$ at frequency $\omega_{0}$ radiate gravitational power

$$
\mathcal{P}=\frac{2}{15} \frac{G}{c^{5}} \mathcal{M}^{2} \ell^{4} \omega_{0}^{6}
$$

Two analogous masses in the fluid radiate sound power

$$
\mathcal{P}=\frac{1}{15 \pi} \frac{1}{v_{s}^{5} M \rho} \mathcal{M}^{2} \ell^{4} \omega_{0}^{8}
$$

These masses are polarons formed around an extremely light impurity, such as an electron in ${ }^{4} \mathrm{H}{ }^{23}$. This result is discussed in more detail in Appendix C.

By analogy, then, the correlation length of gravity need not be the planck length $\xi_{p}=\left(\hbar G / c^{3}\right)^{1 / 2}$.

\section{INTERIOR METRIC}

Let us now consider the implications of this analogy for real gravity. While it is generally impossible to infer the properties of the second phase from measurements made outside the black hole, the simplest guess is that it is literally like the liquid-vapor transition, meaning that the Einstein field equations, like the laws of quantum hydrodynamics, are valid in both phases. Thus we have the following constraints:
1. The equations of classical general relativity outside the black hole are obeyed everywhere except the critical surface.

2. At the critical surface the vacuum of space-time reorganizes itself so as to keep global time defined.

3. The local properties of the vacuum just inside the critical surface are indistinguishable from those just outside.

4. There are no scales other than the mass.

5. The topology is consistent with the collapse of ordinary matter.

The physical indistinguishability of the quantum fluids on either side of a liquid-vapor critical surface is a strong constraint on any gravitational analogue because it requires the relativity principle to operate on both sides. This, in turn, requires that a metric be defined and obey equations something like the Einstein field equations on both sides. The considerations at the critical surface then extrapolate to the entire bulk interior, since an inability to do so would imply a second phase boundary. If the metric exists in the interior of the black hole then one can measure its curvature and compute from this the Einstein tensor. This must be real stress-energy, because if it is not then the space-time has a local property distinguishing it from the space-time outside.

Let us now write these ideas formally. The most general spherically symmetric metric is 24

$$
d s^{2}=e^{\lambda} d r^{2}+r^{2}\left[d \theta^{2}+\sin ^{2}(\theta) d \phi^{2}\right]-e^{\nu} d t^{2} .
$$

The corresponding stress-energy tensor is

$$
\begin{gathered}
R_{11}-\frac{1}{2} g_{11} R=-\nu^{\prime} / r-\left(1-e^{\lambda}\right) / r^{2} \\
R_{22}-\frac{1}{2} g_{22} R=\frac{1}{\sin ^{2}(\theta)}\left[R_{33}-\frac{1}{2} g_{33} R\right] \\
=-r^{2} e^{-\lambda}\left(\frac{\nu^{\prime \prime}}{2}-\frac{\lambda^{\prime} \nu^{\prime}}{4}+\frac{\nu^{\prime 2}}{4}+\frac{\nu^{\prime}-\lambda^{\prime}}{2 r}\right) \\
R_{00}-\frac{1}{2} g_{00} R=e^{\nu-\lambda}\left[-\lambda^{\prime} / r+\left(1-e^{\lambda}\right) / r^{2}\right] .
\end{gathered}
$$

Outside the black hole the Einstein equations require this to be zero, which gives the Schwarzschild solution $\gamma(r)=e^{\nu}=e^{-\lambda}=1-2 \mathcal{M} / r$. The choice of integration constant $2 \mathcal{M}$ determines the location of the event horizon. If we then require the horizon be a critical surface we must also have $e^{\nu}=e^{-\lambda}$ immediately inside the horizon as well, but converging to zero with the opposite slope. This, in turn, requires the presence of matter with negative pressure inside the black hole. $\left(\nu^{\prime}\right.$ and $1-e^{\lambda}$ 
are both negative at the horizon but must limit to zero at the origin.) The matter must also naturally resist falling into the minimum of the gravitational potential, which necessarily lies at the horizon. These properties are so difficult to achieve with any kind of conventional matter the only reasonable choice is a nonzero cosmological constant. Thus inside the black hole we must have

$$
R_{\mu \nu}-\frac{1}{2} g_{\mu \nu} R=\frac{3}{4 \mathcal{M}^{2}} g_{\mu \nu}
$$

where the constant $\mathcal{M}$ is picked to match the boundary condition at $r=2 \mathcal{M}$. This solution has the additional useful feature that the energy inside the black hole sums correctly to $\mathcal{M}$. The result is the metric

$$
\gamma(r)=\left[\begin{array}{ll}
1-(r / 2 \mathcal{M})^{2} & r<2 \mathcal{M} \\
1-2 \mathcal{M} / r & r>2 \mathcal{M}
\end{array}\right]
$$

shown in Fig. 1. It corresponds physically to a vacuum vessel containing a region of space-time with a positive cosmological constant. Note the similarity to Fig. 3.

The singularity at the event horizon corresponds to a negative surface tension or stress required to contain the negative pressure inside the black hole. It may be seen from Eqs. (39) - (41) to show up only in the 22 and 33 components of the Einstein tensor. A balloon with surface tension $\mathcal{T}$ filled gas at pressure $P$ will acquire a radius $r$ satisfying $\mathcal{T}=r P / 2$. Similarly the black hole with local pressure $P=-3 c^{8} / 32 \pi G^{4} \mathcal{M}^{2}$ in proper coordinates inside must have surface tension $\mathcal{T}=-3 c^{2} / 32 \pi G^{2} \mathcal{M}$ at the horizon in proper coordinates. This tension is generated by the space-time itself as it undergoes the transition between its two phases and thus need not be constrained by the properties of any familiar kinds of matter. However, it is actually quite small. To see this let us imagine emulating the stress by generating thermal photons at infinity and allowing them to fall down on the black hole. The light pressure in proper coordinates at the horizon is formally divergent because of the gravitational potential. However, this is false pathology because proper coordinates do not make physical sense at the horizon. Per Fig. 7, the light pressure measured in proper coordinates far away from the black hole is actually finite. The cosmological constant pressure in these same coordinates is zero at the horizon. It is thus always negligible compared to any background thermal radiation pressure.

If the event horizon is indeed a critical surface then its heat capacity measured by distant observers is finite. With $\omega_{q}$ defined as in Eq. (16) with $v_{s}=c \gamma^{1 / 2}(r)$ we have for the the total energy per bosonic degree of freedom inside the event horizon

$$
\begin{aligned}
E & =\int_{0}^{2 G \mathcal{M} / c^{2}}\left[\frac{\gamma^{-1 / 2}(r)}{2 \pi^{2}} \int_{0}^{\infty} q^{2} \frac{\hbar \omega_{q}}{\exp \left(\beta \hbar \omega_{q}\right)-1} d q\right] r^{2} d r \\
& \simeq 1.1 \times\left\{\left[\frac{4 \pi}{3}\left(\frac{2 G \mathcal{M}}{c^{2}}\right)^{3}\right]\left[\frac{\pi^{2}}{30} \frac{\left(k_{B} T\right)^{4}}{(\hbar c)^{3}}\right]\right\} \frac{M c^{2}}{k_{B} T} \cdot \quad(44)
\end{aligned}
$$

Thus the heat content of the black hole is $\sim M c^{2} / k_{B} T$ times the volume of empty space of the same radius. This may also be written $E=7.6 \times 10^{-4} M c^{2}\left(T / T_{H}\right)^{3}$, where $T_{H}=\hbar c^{3} / 8 \pi k_{B} G \mathcal{M}$ is the Hawking temperature.

\section{DISCUSSION}

The resolution of the black-hole paradox we have proposed here conflicts fundamentally with the relativity principle, in that it requires quantum gravity to have a mass scale $M$ that can be measured. If such a scale exists at a black hole horizon then it must exist in asymptotically flat space-time as well and correspond to an absolute velocity scale at which a particle gains mass and loses integrity. This is not so different from the effects of a new elementary particle at this scale, except that decays normally forbidden by relativistic kinematics, i.e. one photon going to three, become possible. No such scale has ever been observed. However the idea that Einstein gravity is emergent in the sense we describe is inherently falsifiable. The relativity principle itself must break down at sufficiently high energy scales, and this breakdown must show up experimentally as spontaneous decay of bosons, such as photons, that otherwise should have integrity. This might have observable effects on the highest-energy cosmic rays.

Our theory also predicts that black holes have specific spectroscopic signatures that can be observed from outside the horizon. By analogy with Fig. 5 we expect the horizon to be highly reflective to light of frequency less than c times the black hole radius and to transmit light slightly above this frequency in resonances that depend on the angle of incidence. In terms of the planck mass $M_{p}=(\hbar c / G)^{1 / 2}=2.18 \times 10^{-5} \mathrm{gm}$ and the mass of the sun $M_{\odot}=2 \times 10^{33} \mathrm{gm}$ we have

$$
\begin{gathered}
\tau_{0}=\frac{2 G \mathcal{M}}{c^{3}}=\left(\frac{\mathcal{M}}{M_{\odot}}\right) \times 1.00 \times 10^{-5} \mathrm{sec} \\
Q_{0}=\sqrt{\frac{2 M}{\hbar \tau_{0}}}=\sqrt{\left(\frac{M}{M_{p}}\right)\left(\frac{M_{\odot}}{\mathcal{M}}\right)} \times 6.47 \times 10^{13} \mathrm{~cm}^{-1} .
\end{gathered}
$$

Thus if $M$ is comparable to the planck mass then the transverse momentum $Q$ of an incoming photon will always be small compared with $Q_{0}$ unless its energy far from the black hole exceeds $\hbar c Q_{0}=1.28 \times 10^{9} \mathrm{eV}$. This implies that the reflectivity of Fig. 5 is a fairly apt description of what one would see for a cold solar-mass black hole. Both the reflection threshold and the transmission resonances would be in the radio near $10^{5} \mathrm{sec}^{-1}$. The reflectance edge is similar to a classicaleffect caused by the convergence of the radial coordinate25, but the resonances have no classical analogue. By analogy with the inelastic scattering of high-frequency sound from a critical surface, we expect that high-frequence electromagnetic radiation will be inelastically backscattered from 
the event horizon with a characteristic spectrum terminating at a red shift of $90 \%$ for normal incidence. Exactly how high the energy must be for this process to be efficient depends on the matrix element for decay. If we assume the latter to be set by planck units also, then we have $\tau_{0} / \tau \sim\left(E / \hbar c Q_{0}\right)^{2}$. This implies that the process is efficient only for hard gamma rays, and that most photons with energies less than $10^{9} \mathrm{eV}$ pass through the horizon without decaying. Once on the other side they refract away from the center of the black hole, as in a defocussing lens.

We also expect the black hole horizon to be a thermalizer of radiation and to be itself a thermal body with a finite positive heat capacity measured by an observer at infinity. This heat capacity is comfortably small for astrophysical objects. Rewriting Eq. (44) as

$$
\frac{E}{\mathcal{M} c^{2}}=3.24 \times 10^{-20}\left(\frac{M}{M_{p}}\right)\left(\frac{\mathcal{M}}{M_{\odot}}\right)^{2}\left(\frac{T}{1^{\circ} K}\right)^{3}
$$

we that that if $M$ is the planck mass then the heat content of a solar-mass black hole becomes comparable to its mass when $T \sim 10^{6}{ }^{\circ} \mathrm{K}$. This implies that the temperature of a solar-mass black hole might well be sufficiently high $\left(>10^{3}{ }^{\circ} \mathrm{K}\right)$ to make it visible against the cosmic microwave background.

The specific metric we propose identifies the second phase as de Sitter space and its distinguishing characteristic as a nonzero cosmological constant. However, it is arguable that the key distinguishing characteristic is not the cosmological constant, per se, but topology. The cosmological constant we find depends on the black hole mass and becomes unmeasurably small when the latter is large. The horizon is effectively planar in this limit, and the two phases locally indistinguishable. However, one can see from Figs. (2) and (3) that exactly the same thing occurs at the liquid-vapor critical point. The liquid and vapor sides of the transition are distinguished only in how the critical equation of state eventually deviates from symmetric inflection, which is a global property. However, one can imagine resolving this problem by eliminating the earth's gravitational field in Fig. 3 and substituting the field due to self-gravitation of the fluid. Then "down" is determined by the center of gravity of the fluid, the critical surface is a sphere, and the two phases are distinguished as a practical matter by which is inside the sphere and which outside.

While we cannot rule out on any technical grounds the possibility that the transition is first-order, we find it highly unlikely because it would require the the metric to be discontinuous. Referring to Fig. 2, we see that perturbing the critical equation of state downward causes the susceptibility of the soft excitation - in this case sound - to become negative, so that density perturbations of the uniform state grow. The uniform state is thus absolutely unstable to a nonuniform one characterized by position-dependent density. The relevant soft excitation in Einstein gravity is a gravity wave

$$
\delta g_{x x}=-\delta g_{y y} \sim e^{i(k z-\omega t)}
$$

If this excitation were to become unstable in the same way it would generate a nonuniform metric with sharp jumps analogous to the density jumps at liquid-vapor interfaces discussed in Appendix A. This would be a much more violent breakdown of classical general relativity, and in particular could not be interpreted as interface stress-energy.

Regardless of whether it the event horizon corresponds to a first- or second-order phase transition, identifying the space-time of a black hole as quantum ground state resolves the information paradox 26. The horizon does not destroy quantum information but rather makes entropy the same way black paint does, i.e. by scattering the energy into a thermodynamically large number of degrees of freedom. This is conceptually similar to the quantum holography ideas27, except that the relevant degrees of freedom are collective in nature rather than fundamental. Also, we find that the that vacuum beyond the horizon is locally identical to the one we know and can be probed experimentally from the outside. Insofar as string theory predicts something else, the two theories can be distinguished from each other by experiment.

Our picture for black hole is also fundamentally different from the classical one, in that we find quantum effects determine both the nature of the event horizon and the interior spacetime, even in the case of macroscopic black holes. The possibilities for falsifying our predictions and thereby demonstrating the quantum nature of black holes is, of course, most exciting.

\section{ACKNOWLEDGEMENTS}

This work was supported primarily by the National Science Foundation under Grant DMR-9813899. Additional support was provided by NASA under Collaborative Agreement No. NCC 2-794.

\section{APPENDIX A: MAXWELL CONSTRUCTION}

The Maxwell construction of the zero-temperature equation of state shown in Fig. 2 comes from the condition that the superfluid order parameter be extremal across the liquid-vapor interface. As is the case at finite temperature the end points of the Maxwell loops have identical pressures $P$ and chemical potentials:

$$
\begin{gathered}
\mathcal{U}\left(\psi_{1}^{2}\right)-\mu \psi_{1}^{2}=\mathcal{U}\left(\psi_{2}^{2}\right)-\mu \psi_{2}^{2}=-P \\
\mathcal{U}^{\prime}\left(\psi_{1}^{2}\right)=\mathcal{U}^{\prime}\left(\psi_{2}^{2}\right)=\mu .
\end{gathered}
$$

Let us now solve

$$
-\frac{\hbar^{2}}{2 M} \frac{d^{2} \psi}{d z^{2}}+\left[\mathcal{U}^{\prime}\left(\psi^{2}\right)-\mu\right] \psi=0
$$


across the interface. Multiplying both sides of the equation by $d \psi / d z$ we obtain

$$
\begin{aligned}
& \frac{d}{d z}\left\{-\frac{\hbar^{2}}{2 M}\left(\frac{d \psi}{d z}\right)^{2}+\mathcal{U}\left(\psi^{2}\right)-\mu \psi^{2}\right\}=0 \\
& -\frac{\hbar^{2}}{2 M}\left(\frac{d \psi}{d z}\right)^{2}+\mathcal{U}\left(\psi^{2}\right)-\mu \psi^{2}+P=0 .
\end{aligned}
$$

Integrating this by quadratures we then generate the density profile shown in Fig. 8. Eqs. (A1) and A2 guarantee that the profile approaches $\psi_{1}$ and $\psi_{2}$ asymptotically. Deep in phase 1 we have, with $\psi=\psi_{1}+\delta \psi$,

$$
\mathcal{U}(\psi)-\mu \psi^{2} \simeq \frac{B_{1}}{2 \rho_{1}} \delta \psi^{2}
$$

and thus

$$
\pm \xi_{1} \int \frac{d(\delta \psi)}{\delta \psi} \simeq \int d z \quad \psi-\psi_{1} \sim e^{ \pm z / \xi_{1}}
$$

The sign is picked to make $\delta \psi$ vanish away from the interface. Doing this for side 2 as well, we see that the thickness of the interface region is essentially $\xi_{1}+\xi_{2}$ and diverges at the critical point.

The surface tension is obtained by computing the total energy and subtracting off the energy one would obtain in the limit of $\hbar \rightarrow 0$ or $M \rightarrow \infty$, when the interface becomes sharp and the energy comes entirely from $\mathcal{U}\left(\psi_{1}^{2}\right)$ and $\mathcal{U}\left(\psi_{2}^{2}\right)$. Let $\psi_{0}$ denote this solution. Then the chemical potential $\mu$ must be the same for $\psi_{0}$ as it is for $\psi$ since the values of the two much match far away from the interface. The pressure $P$ is also be the same because neither $\psi_{0}$ nor $\psi$ has a gradient away from the interface. We also have

$$
\mu \int\left(\psi^{2}-\psi_{0}^{2}\right) d z=0
$$

because the number of particles in the two states is the same. Now using Eq. (A5) to eliminate $\mathcal{U}$ from the expression for the energy difference, we obtain

$$
\mathcal{T}=\frac{\hbar^{2}}{M} \int\left(\frac{\partial \psi}{\partial z}\right)^{2} d z
$$

Thus the surface tension is twice the kinetic energy per unit area.

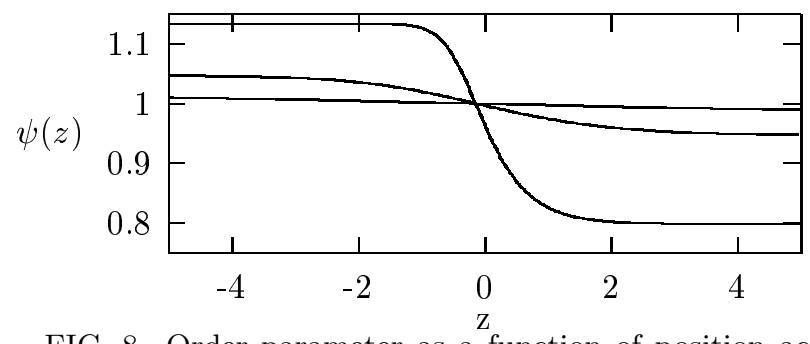

FIG. 8. Order parameter as a function of position across the liquid-vapor interface for the equation of state of Eq. (6) with the values $27 \mathrm{bc} / 8 \mathrm{a}=0.9,0.99$, and 0.999 . As the critical value 1 is approached the density jump across the interface collapses to zero and interface width diverges.

\section{APPENDIX B: REFLECTION COEFFICIENT}

With momentum $Q$ in the plane of the interface, Eq. (30) becomes

$$
\omega^{2} \phi=\left(-\frac{\partial}{\partial z} z^{2} \frac{\partial}{\partial z}+z^{2} Q^{2}\right) \phi+\left(-\frac{\partial^{2}}{\partial z^{2}}+Q^{2}\right)^{2} \phi .
$$

We eliminate the solutions of this equation that diverge as $\exp \left(z^{2} / 2\right)$ by requiring $\phi(z)$ to have a fourier transform. This satisfies

$$
\omega^{2} \hat{\phi}=\left[-\frac{\partial}{\partial q}\left(q^{2}+Q^{2}\right) \frac{\partial}{\partial q}+\left(q^{2}+Q^{2}\right)^{2}\right] \hat{\phi} .
$$

For $Q \neq 0$ this equation is regular at the origin and is easily solved numerically. The first 10 energy eigenfunctions for the case of $Q=10^{-5}$ are shown in Fig. 9. As $Q$ becomes smaller and smaller the nodes of the wavefunction are pulled into the origin. In the $Q \rightarrow 0$ limit the equation becomes singular at $q=0$ and is no longer required to be analytic there. Thus we consider a wavefunction that is zero for $q<0$ and $\hat{\phi}=\sum_{n=0}^{\infty} a_{n} q^{n+\nu}$ for $q>0$. This satisfies the equation when

$$
\nu=-\frac{1}{2} \pm \sqrt{1 / 4-\omega^{2}} \quad \frac{a_{n}}{a_{n-4}}=\frac{1}{n(n+2 \nu+1)} .
$$

For $\omega<1 / 2$ there is no normalizable solution. For $\omega>$ $1 / 2$ on the other hand, there is always one normalizable solution formed by subtracting the expressions for the two allowed values of $\nu$, namely

$$
\hat{\phi}=\left(\frac{2}{q^{2}}\right)^{1 / 4}\left[I_{\left(2 \nu_{1}+1\right) / 4}\left(q^{2} / 2\right)-I_{\left(2 \nu_{2}+1\right) / 4}\left(q^{2} / 2\right)\right] .
$$

Only the behavior of this function near $q \rightarrow 0$ is needed for computing the reflectivity. For large positive $z$ we have

$$
\begin{gathered}
\phi(z) \simeq \int_{0}^{\infty}\left[\left(\frac{q^{2}}{2}\right)^{\nu_{1} / 2}-\left(\frac{q^{2}}{2}\right)^{\nu_{2} / 2}\right] e^{i q z} d q \\
=2^{-\nu_{1} / 2} \Gamma\left(\nu_{1}+1\right)|z|^{1-\nu_{1}} e^{i \pi \nu_{1} / 2}-(1 \rightarrow 2) .
\end{gathered}
$$

For negative $\mathrm{z}$ we have $\phi(z)=-\phi^{*}(-z)$. The real and imaginary parts of $\phi(z)$ are separately solutions, and we may combine them to make a wave with no incoming component on the right side of the barrier. ¿From this we obtain the reflection and transmission coefficients

$$
\begin{aligned}
& T=i 2^{i \alpha} \frac{\Gamma(1 / 2+i \alpha)}{\Gamma(1 / 2-i \alpha)} \tanh (\pi \alpha) \\
& R=2^{i \alpha} \frac{\Gamma(1 / 2+i \alpha)}{\Gamma(1 / 2-i \alpha)} \operatorname{sech}(\pi \alpha),
\end{aligned}
$$

where $\alpha=\sqrt{\omega^{2}-1 / 4}$. 

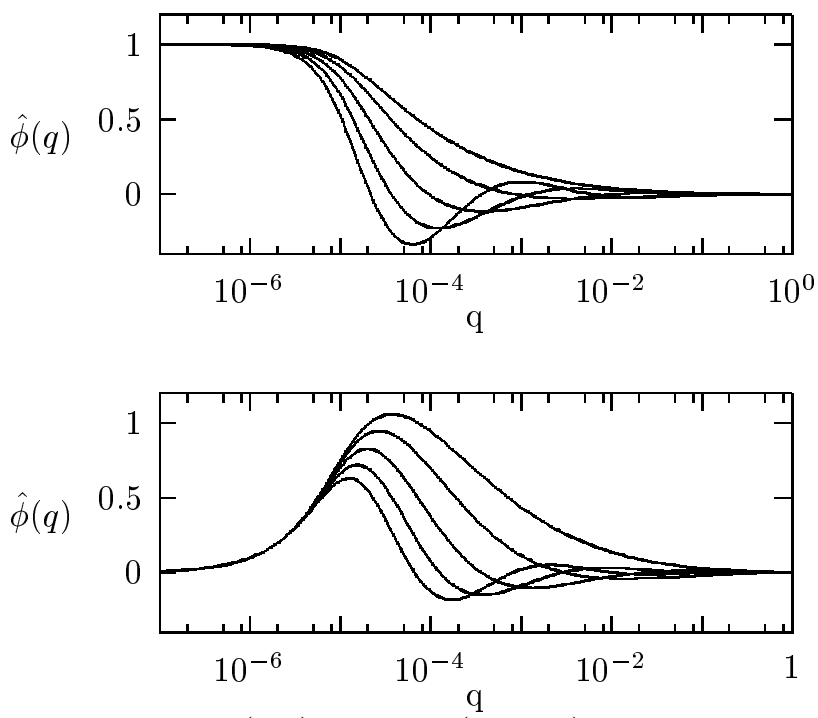

FIG. 9. Even (top) and odd (bottom) energy eigenstates of Eq. (B2) for the case of $Q=10^{-5}$. The corresponding eigenvalue are shown in Fig. 5.

The resonant reflection spectrum one would actually measure is quite sensitive to details of the experiment and cannot be computed without further assumptions. For the purposes of constructing Fig. 5 we assumed a simple barrier with tunneling matrix elements increasing slowly with the number of nodes in the wavefunction and a constant density of states at infinity. Denoting this density of states, times the square of the scale of the tunneling matrix elements, by $t$, we have

$$
|R|^{2}=1-\left|\operatorname{Tr}\left[t G^{0}\left(1+i t G^{0}\right)^{-1}\right]\right|^{2},
$$

where

$$
\begin{gathered}
G_{11}^{0}=G_{22}^{0}=\sum_{n}^{N} \frac{\sqrt{j+1}}{\left(\omega-\omega_{n}+i \eta\right)} \\
G_{12}^{0}=G_{21}^{0}=\sum_{n}^{N}(-1)^{n} \frac{\sqrt{j+1}}{\left(\omega-\omega_{n}+i \eta\right)} .
\end{gathered}
$$

Fig. 5 was generated using $t=0.01$ and $N=20$.

\section{APPENDIX C: POLARON RADIATION}

The linearized equations of motion for $\psi=\psi_{0}+\delta \psi_{\mathrm{R}}+$ $i \delta \psi_{\mathrm{I}}$ in the presence of a weak potential $V(\mathbf{r}, t)$ are

$$
\begin{gathered}
\hbar \frac{\partial\left(\delta \psi_{R}\right)}{\partial t}=-\frac{\hbar^{2}}{2 M} \nabla^{2}\left(\delta \psi_{\mathrm{I}}\right) \\
-\hbar \frac{\partial\left(\delta \psi_{I}\right)}{d t}=-\frac{\hbar^{2}}{2 M} \nabla^{2}\left(\delta \psi_{\mathrm{R}}\right)+\frac{2 B}{\rho}\left(\delta \psi_{\mathrm{R}}\right)+V \psi_{0} .
\end{gathered}
$$

The corresponding classical Hamiltonian is

$$
\begin{aligned}
& \mathcal{H}=\int\left\{V(\mathbf{r}, t)\left(\psi_{0}^{2}+2 \psi_{0} \delta \psi_{\mathrm{R}}\right)+\frac{2 B}{\rho}\left(\delta \psi_{\mathrm{R}}\right)^{2}\right. \\
& \left.+\frac{\hbar^{2}}{2 M}\left(\left|\nabla\left(\delta \psi_{\mathrm{R}}\right)\right|^{2}+\left|\nabla\left(\delta \psi_{\mathrm{I}}\right)\right|^{2}\right)\right\} d \mathbf{r} .
\end{aligned}
$$

Fourier transforming the equations of motion we obtain

$$
\begin{gathered}
-i \hbar \omega \delta \hat{\psi}_{\mathrm{R}}-\frac{\hbar^{2} q^{2}}{2 M} \delta \hat{\psi}_{\mathrm{I}}=0 \\
\left(\frac{\hbar^{2} q^{2}}{2 M}+\frac{2 B}{\rho}\right) \delta \hat{\pi}_{\mathrm{R}}-i \hbar \omega \delta \hat{\psi}_{\mathrm{I}}=\hat{V} \psi_{0}
\end{gathered}
$$

For the static potential $V(\mathbf{r}, t)=V_{0} a^{3} \delta^{3}(\mathbf{r})$ we have $\hat{V}=$ $2 \pi V_{0} a^{3} \delta(\omega)$, which gives $\delta \psi_{\mathrm{I}}=0$ and

$$
\begin{gathered}
\delta \psi_{\mathrm{R}}(\mathbf{r})=-\frac{V_{0} a^{3} \psi_{0}}{(2 \pi)^{3}} \int \frac{1}{\hbar^{2} q^{2} / 2 M+2 B / \rho} e^{i \mathbf{q} \cdot \mathbf{r}} d \mathbf{r} \\
=-V_{0} a^{3} \psi_{0} \frac{2 M}{\hbar^{2}} \frac{e^{-2 r / \xi}}{r} \quad\left(\xi=\frac{\hbar}{M v_{s}}\right) .
\end{gathered}
$$

The amount of fluid accumulated is thus

$$
\mathcal{M}=M \int 2 \psi_{0} \delta \psi_{\mathrm{R}} d \mathbf{r}=-\frac{V_{0} a^{3} \rho}{v_{s}^{2}} .
$$

For the time-dependent potential

$$
V(\mathbf{r}, t)=V_{0} a^{3}\left\{\delta^{3}\left[\mathbf{r}-\mathbf{r}_{0}(t)\right]+\delta^{3}\left[\mathbf{r}+\mathbf{r}_{0}(t)\right]\right\},
$$

where $\mathbf{r}_{0}(t)=(\ell / 2)\left[\cos \left(\omega_{0} t\right), \sin \left(\omega_{0} t\right), 0\right]$ and $\omega_{0} \ell<<v_{s}$, only the quadrupole terms survive in the far field, and we have

$$
\begin{aligned}
& \delta \psi(r, \theta, \phi) \simeq \frac{V_{0} a^{3} \psi_{0} \ell^{2} \omega_{0}^{3} \sin ^{2}(\theta)}{8 \pi \hbar v_{s}^{4} r} \\
& \times\left[\frac{4 \hbar \omega_{0}}{M v_{s}^{2}} \cos (\chi)+i \sin (\chi)\right],
\end{aligned}
$$

where $\chi=2 \omega_{0}\left(r / v_{s}-t\right)-\phi$. The radiated energy flux is then

$$
\frac{d \mathcal{P}}{d \Omega} \simeq \frac{\left(V_{0} a^{3} \psi_{0} \ell^{2} \omega_{0}^{4}\right)^{2}}{32 \pi^{2} M v_{s}^{9}} \sin ^{4}(\theta)
$$

Combining this with Eq. (C7) we obtain finally

$$
\mathcal{P} \simeq \frac{\left(\mathcal{M} \ell^{2} \omega_{0}^{3}\right)^{2}}{M \rho v_{s}^{5}} \frac{1}{16 \pi} \int_{-1}^{1}\left(1-\mu^{2}\right)^{2} d \mu
$$

per Eq. (37). 
${ }^{1}$ G. 'tHooft, Class. Quant. Grav. 16, 3263 (1999).

${ }^{2}$ S. Hawking, Phys. Rev. D 14, 2460 (1976)

${ }^{3}$ G. E. Volovik, "Superfluid Analogies of Cosmological Phenomena," Phys. Rep. (in press) gr-qc/0005091.

${ }^{4}$ A. Sakharov, Sov. Phys. Dok. 12, 1040 (1968).

${ }^{5}$ G. Chapline, Mod. Phys. Lett. A 7, 1959, (1992).

${ }^{6}$ W. G. Unruh, Phys. Rev. Lett. 46, 1351 (1981).

${ }^{7}$ T. A. Jackobson and G. E. Volovik, Phys. Rev. D 58, 064021; ibid., "Effective Spacetime and Hawking Radiation from Moving Domain Wall in Thin Film of ${ }^{3} \mathrm{He}-\mathrm{A}$ ", gr-gc/9811014; G. E.Volovik, JETP Lett. 69, 705 (1999).

${ }^{8}$ L. J. Garay, J. R. Anglin, J. I. Cirac, and P. Zoller, Phys. Rev. Lett. 85, 4643 (2000).

9 M. Mohazzab, "Second Sound Horizon," J. Low Temp. Phys. (in press).

${ }^{10}$ M. Ueda and K. Huang, Phys. Rev. A 60, 3317 (1999);

A. Eleftheriou and K. Huang, "Instability of a BoseEinstein Condensate with Attractive Interaction," condmat/9908229

${ }^{11}$ M. R. Andrews et al., Phys. Rev. Lett 79, 553 (1997).

${ }^{12}$ H. R. Glyde, Excitations of Liquid and Solid Helium, (Oxford U. Press, New York, 1994).

${ }^{13}$ N. N. Bogoliubov, J. Phys. U.S.S.R. 11, 23 (1947).

14 M. D. Miller, L. H. Nosanow, and L. J. Parish, Phys. Rev. B. 15, 214 (1977).

${ }^{15}$ A. Gammal, T. Frederico, L. Tomio, and Ph. Chomaz, Phys. Rev. A 61, 051602(R) (2000).

16 S. Inouye et al., Nature 392, 151 (1998); J. Stenger et al., Phys. Rev. Lett. 82, 2422 (1999).

17 S. L. Cornish et al., Phys. Rev. Lett. 85, 11795 (2000).

${ }^{18}$ K. Huang, Statistical Mechanics (Wiley, New York, 1963), p. 40.

${ }^{19}$ V. L. Ginzburg and L. P. Pitaevskii, Zh. Eksp. Teor. Fiz. 34, 1240 (1958) [Sov. Phys. JETP 7, 858 (1958)]; L. V. Pitaevskii, ibid. 40, 646 (1961) [ ibid. 13, 451 (1961)]; E. P. Gross, J. Math. Phys. 4, 195 (1963).

${ }^{20}$ L. Landau, J. Phys. U.S.S.R 5, 71 (1941); I. M. Khlalatnikov, An Introduction to the Theory of Superfluidity, (Benjamin, New York, 1965), p. 53.

${ }^{21}$ H. E. Stanley, Introduction to Phase Transitions and Critical Phenomena, (Oxford. U. Press, New York, 1971); C. Domb, A Historical Introduction to the Modern Theory of Critical Phenomena, (Taylor and Francis, Bristol, PA, 1996).

22 S. Sachdev, T. Senthil, and R. Shankar, Phys. Rev. B 50, 258 (1994); S. Sachdev, Quantum Phase Transitions (Cambridge U. Press, Cambridge, 1999), p 214.

${ }^{23}$ C. C. Grimes and G. Adams, Phys. Rev. B 45, 2035 (1992), and references therein.

${ }^{24}$ A. S. Eddington, The Mathematical Theory of Relativity (Cambridge U. Press, London, 1965).

${ }^{25}$ R. H. Price, Phys. Rev. D, 2419 (1972).

${ }^{26}$ G. Chapline, in Foundations of Quantum Mechanics, ed. by T. D. Black et al. (World Sci., Singapore, 1992).

${ }^{27}$ L. Susskind, Phys. Rev. Lett. 71, 2367 (1993). 